\title{
Fine steering mirror based on piezo actuators: a point ahead mechanism (PAM30) for deep space optical communication module of the psyche mission
}

Sandrine Hugi, Thomas Maillard, Anthony Baillus, Gérald Aigouy, Olivier Sosnicki, et al.

Sandrine Hugi, Thomas Maillard, Anthony Baillus, Gérald Aigouy, Olivier Sosnicki, Francois Barillot, "Fine steering mirror based on piezo actuators: a point ahead mechanism (PAM30) for deep space optical communication module of the psyche mission," Proc. SPIE 11852, International Conference on Space Optics - ICSO 2020, 118525N (11 June 2021); doi:

10.1117/12.2599966

SPIE Event: International Conference on Space Optics - ICSO 2021, 2021, Online Only 


\section{International Conference on Space Optics-ICSO 2020}

Virtual Conference

30 March-2 April 2021

Edited by Bruno Cugny, Zoran Sodnik, and Nikos Karafolas
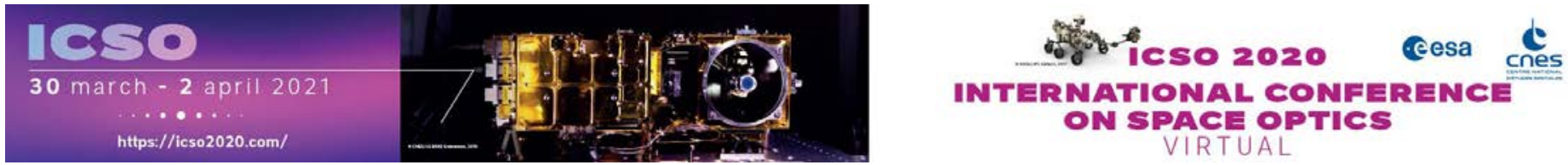

FINE STEERING MIRROR BASED ON PIEZO ACTUATORS:
POINT AHEAD MECHANISM (PAM30) FOR DEEP SPACE
OPTICAL COMMUNICATION MODULE OF THE PSYCHE
MISSION

Cesa isoporesedings ecres

International Conference on Space Optics - ICSO 2020, edited by Bruno Cugny, Zoran Sodnik, Nikos Karafolas, Proc. of SPIE Vol. 11852, 118525N · (c) 2021 ESA and CNES CCC code: $0277-786 \mathrm{X} / 21 / \$ 21 \cdot$ doi: $10.1117 / 12.2599966$ 


\title{
FINE STEERING MIRROR BASED ON PIEZO ACTUATORS: A POINT AHEAD MECHANISM (PAM30) FOR DEEP SPACE OPTICAL COMMUNICATION MODULE OF THE PSYCHE MISSION
}

\author{
Adrien Guignabert, Thomas Maillard, Anthony Baillus, Gerald Aigouy*, Olivier Sosnicki and \\ Francois Barillot
}

CEDRAT TECHNOLOGIES SA

\begin{abstract}
The purpose of this paper is to present the development of a new dual axis (tip-tilt) mechanism, with integrated $\mathrm{SiC}$ mirror, designed for the Jet Propulsion Laboratory (JPL) and integrated inside their Deep Space Optical Communication (DSOC) module of incoming Psyche mission to be launched in 2022. This paper presents the design, assembly and tests of the Engineering (EM), Qualified (QM) and Flight (FM) models. Regarding the design phase, an emphasis is put on the mirror design and computations to ensure that the required flatness would be maintained after the integration and the part would withstand the thermal/mechanical environment. The optical measurement performed after assembly is also presented. The qualification results for a new alpha-case removal process for titanium parts are presented. Test results are especially interesting regarding the temperature behavior of the mechanism, and impact on the stroke and strain gage sensors' feedback.
\end{abstract}

\section{INTRODUCTION}

In the incoming NASA Psyche mission, JPL is planning the assessment of a first Deep Space Optical Communication (DSOC) module. In this module, a Point Ahead Mechanism (PAM30) aims at steering the optical downlink signal towards anticipated earth position during DSOC phases.

As a background, for 20 years, Cedrat Technologies (CTEC) has provided various piezoelectrically-actuated Beam Steering Mirrors (BSM) as well as Fast Steering Mirrors (FSM) for space missions (PHARAO for CNES, ATLID for Airbus DS) active in Free Space Or

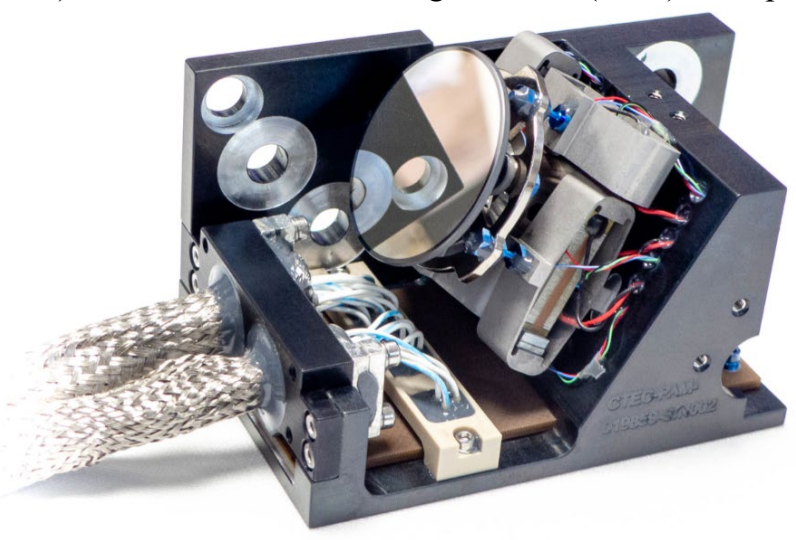

Figure 1: PAM30 EQM picture after assembly

I, CTEC has also been 
In this context, CTEC was sub-contracted by L3-Harris, to design, manufacture and test the performances of the PAM30 engineering and flight models for JPL PSYCHE DSOC. The developed PAM30 is new tip-tilt mechanism based on low voltage Amplified Piezoelectric Actuators APA ${ }^{\circ}$, exploiting its space heritage.

This paper presents the design, assembly and tests of the produced PAM30 models, covering the following parts and failure modes: piezo materials for actuation, strain gages for indirect angular position sensing, mechanical parts treatments, tip-tilt mechanical structure, mirror flatness...

\section{PAM30 DESIGN}

\subsection{Specifications and timeline}

The main specifications for this mechanism were to ensure an angular stroke of $+/-2.8$ mrad throughout the full operational temperature range of the mission $\left(-25 /+50^{\circ} \mathrm{C}\right.$ full perf, $-40 /+65^{\circ} \mathrm{C}$ reduced perf $)$ and a mirror surface flatness under $63 \mathrm{~nm}$ while remaining inside a very limited volume and surviving launch vibrations.

The project really started in summer 2018 with a preliminary feasibility study aimed at checking that these specifications could be reached, which ended positively in Fall 2018. The initial design was first based on ATLID mechanism as a building block but specific requirements made it progressively noticeably different in the end. The required schedule for the final mechanism development, i.e. delivering Flight Models in less than a year after the actual project start, was very unusual and challenging.

\subsection{Mechanism overview}

The piezo actuators are cabled in 2 push-pull configurations (1 per axis) to allow a direct mirror rotation control.

The PAM itself is composed of the following parts:

- $\quad$ A bracket baseplate (in aluminum): The APA ${ }^{\circledR}$ are fixed on it with screws.

- $\quad 4 \mathrm{APA}{ }^{\circledR}$ (in titanium): They provide the required displacement and are fixed to the baseplate and to the mirror support via flexural pivots. The APA ${ }^{\circledR}$ are equipped with $\mathrm{SG}$ sensors by gluing process.

- 4 circular pivots (in titanium).

- A mirror support (in INVAR) which holds the mirror.

- A guiding blade (in titanium) soldered onto the central cylinder that stiffens the assembly.

- A polished Silicon Carbide (SiC substrate) based mirror supplied by Mersen OptoSiC ${ }^{\circ}$ and coated by Materion.

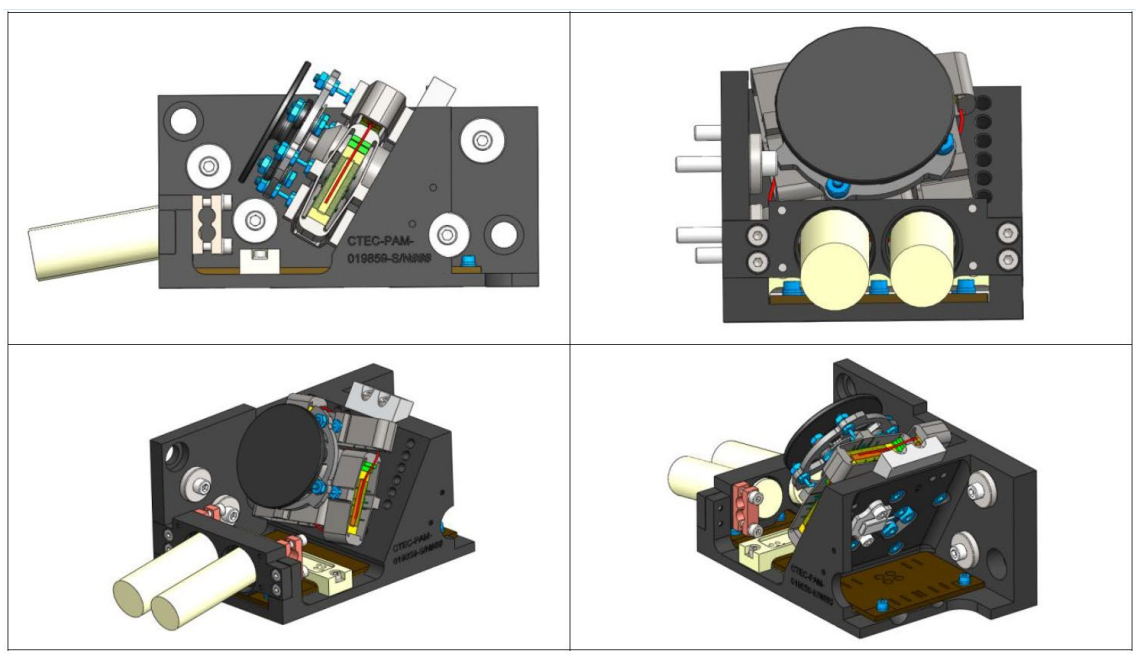

Figure 2: PAM30 CAD overview from Cedrat Technologies 


\subsection{Strain gauge (SG) sensing}

To monitor the mirror angle, an indirect solution using strain gages (SG) placed on each piezo actuator is selected. This SG sensor monitoring solution benefits from a space heritage from other projects, especially ATLID on this matter, which enabled an important development on the SG assembly process. The initial SG redundancy requirement was lifted because it led to an important wiring complexity (32 instead of 16 wires) amongst other additional constraints.

The project used constantan, $350 \mathrm{Ohm}$ SG. There are $2 \mathrm{SG}$ per piezo stack, mounted in one full Wheatstone bridge per rotation axis to maximize the sensitivity while minimizing thermal drift. All SG wires and PCB traces are the same length to limit offset drift.

\subsection{New amplified piezoelectric actuator design}

The existing CTEC actuators were either slightly too short in stroke or not stiff enough to ensure the mechanism survival during the launch. The mechanism is composed of 4 Amplified Piezo Actuators (APA $\left.{ }^{\circledR}\right)$, deriving from CTEC standard APA120S but specifically designed for the application needs.

Based on CTEC space heritage, the APA® shell was made in Ti6Al4V titanium, allowing a theoretical infinite fatigue lifetime in the specified operational conditions and an interesting stiffness/mass ratio. Another interest of the use of titanium rather than steel as used in standard products, is the limitation of the thermal stroke effect of such amplified actuator, given the titanium closer coefficient of thermal expansion $(9 \mathrm{ppm} / \mathrm{K})$ to the piezo stack (typically -3 to $+1 \mathrm{ppm} / \mathrm{K})$ compared to a high-performance stainless steel for example $(10-11 \mathrm{ppm} / \mathrm{K})$.

A total of 18 APA ${ }^{\circledR}$ were assembled and tested, the results are indicated in the following table:

\begin{tabular}{|l|c|c|}
\hline & $\begin{array}{c}\text { Full stroke } \\
(\mathbf{1 7 0 V p p )}\end{array}$ & $\begin{array}{c}\text { 1st coupled resonant } \\
\text { frequency }\end{array}$ \\
\hline Average measured & $\mu \mathrm{m}$ & $\mathrm{Hz}$ \\
\hline Standard deviation measured & 149.2 & 6963.6 \\
\hline Design value (worst case) & 2.0 & 60.9 \\
\hline Difference measurement/design value & 130.4 & 6151 \\
\hline
\end{tabular}

Table 1: PAM actuators measurements results

The design values are considering worst cases, i.e. low piezo gain and stiffest shell regarding the stroke. A conservative approach was considered to ensure that the required mechanism stroke would be reached and as always, we ended up with a stroke/stiffness average margin of 14 and $13 \%$.

\section{INTEGRATED OPTICS - SIC MIRROR DEVELOPMENT}

The PAM is designed around one of its core components: the mirror. For this new project, CTEC has used its experience on piezo-optical aerospace projects and collaborated with partners in order to fully integrate the optical part development, from the $\mathrm{SiC}$ mirror design to its integration and optical verifications. 


\subsection{SiC Mirror design}

One of the main design constraints of an embedded optics mechanism is to minimise the mirror surface deformation and to keep the induced optical wave front error below the requirements. On this case, a maximum of $63 \mathrm{~nm}$ mirror surface flatness is the target. To ensure the specification would be reached, CTEC developed tools and performed specific simulations in the early design phase. Specifically including verifications of induced surface deformation caused by mechanical biases, thermal deformation as well as optimisation of mirror shape and dimensions.
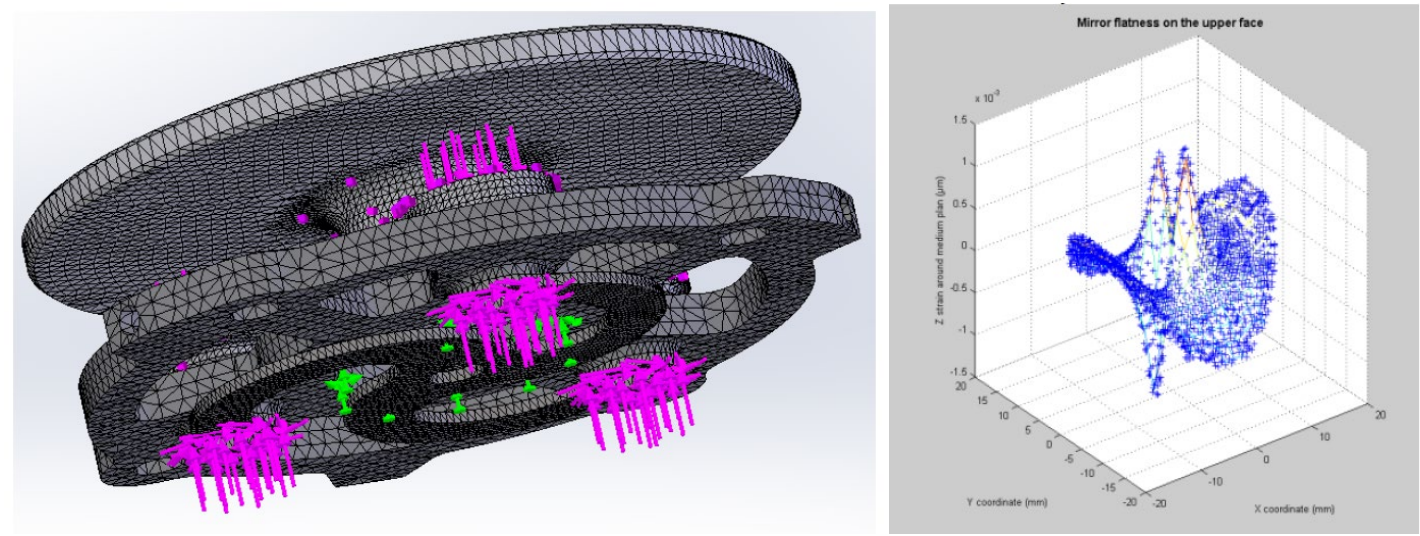

Figure 3: Mirror surface deformation simulation meshing and boundary conditions (screw tension and torque case) (right) - Mirror surface deformation evaluation results (left)

The mirror design process is based on a finite element simulation of each mirror deformation contributor case: mirror clamping to its support, actuator height variation, screw tightening, thermal operational. Each case uses representative boundary conditions. The resulting displacement on each of the mirror surface points is then exported. A Matlab program is then run to specifically post process the results from the simulation. Extracting the RMS reference plane from this data set, it calculates the distance of each point to this plane, the peaks and valley of the deformed mirror and the resulting RMS deformation. The specific contribution of each evaluated case on the mirror deformation is then summed up to give an estimation of the total expected mirror surface deformation.

PAM development also included a regular mirror optical verification at different stages of the assembly, with the intention of having the ability to stop the process in case a mirror would appear out of the acceptable range or showing important surface evolution from one step to another. With the recent CTEC experience on this matter, such regular controls also allowed us to learn a lot regarding the impact of each assembly step as well as providing a safer project assembly process.
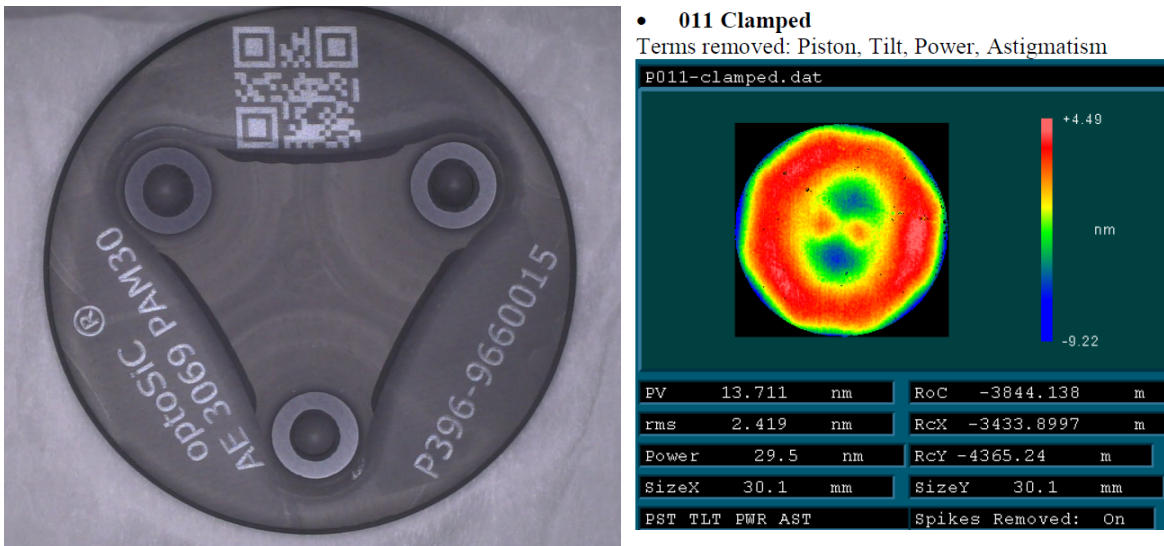

Figure 4: Mirror interface (left) and surface verification with interferometer (right) 


\subsection{Mirror verification results}

A total of 4 PAM30 were produced and the mirror Reflected Wavefront Error (RWE), Peak-to-Valley (RWE PV, basically 2 times mirror surface flatness error SFE) was controlled in 3 different boundary conditions, i.e. the coated mirror in the initial free condition, clamped on its mount and integrated in the mechanism.

\begin{tabular}{|c|c|c|c|c|c|c|c|c|}
\hline \multirow[t]{2}{*}{ PAM model } & \multicolumn{2}{|c|}{ 1.Free coated mirror } & \multicolumn{2}{|c|}{$\begin{array}{l}\text { 2. Mirror clamped on } \\
\text { mount }\end{array}$} & \multicolumn{2}{|c|}{$\begin{array}{l}\text { 3.Mirror integrated } \\
\text { in final mechanism }\end{array}$} & \multicolumn{2}{|c|}{$\begin{array}{l}\text { Calculation: Mechanism } \\
\text { contribution to mirror } \\
\text { deformation }\end{array}$} \\
\hline & $\begin{array}{c}\text { RWE } \\
\text { PV (nm) }\end{array}$ & $\begin{array}{l}\text { RWE } \\
\text { RMS } \\
(\mathrm{nm})\end{array}$ & $\begin{array}{l}\text { RWE PV } \\
(\mathrm{nm})\end{array}$ & $\begin{array}{l}\text { RWE } \\
\text { RMS } \\
(\mathrm{nm})\end{array}$ & $\begin{array}{c}\text { RWE } \\
\text { PV (nm) }\end{array}$ & $\begin{array}{l}\text { RWE } \\
\text { RMS } \\
(\mathrm{nm})\end{array}$ & $\begin{array}{l}\text { RWE PV } \\
(\mathrm{nm})\end{array}$ & $\begin{array}{l}\text { RWE RMS } \\
\text { (nm) }\end{array}$ \\
\hline EM & 38.1 & 10.1 & 34.9 & 8.9 & 41.3 & 10.6 & 3.2 & 0.5 \\
\hline EQM & 22.2 & 6.2 & 27.2 & 6.6 & 38.7 & 10.3 & 16.5 & 4.1 \\
\hline FM1 & 23.2 & 5.5 & 19.4 & 4.6 & 26.6 & 6.0 & 3.4 & 0.5 \\
\hline FM2 & 28.9 & 8.1 & 33.8 & 8.2 & 34.1 & 9.5 & 5.2 & 1.4 \\
\hline Average & 28.1 & 7.5 & 28.8 & 7.1 & 35.2 & 9.1 & 7.1 & 1.6 \\
\hline
\end{tabular}

Table 2: PAM mirror surface flatness measurement results

With an average $35.2 \mathrm{~nm}$ and a maximum of 41.3 RWE PV, the flatness specification "< $127 \mathrm{~nm}$ RWE" is reached with significant margins. The last column evaluates the proper mechanism contribution to mirror deformation based on the difference between the final and the initial RWE PV measurements.

The mechanism proper contribution to the mirror deformation appears to be limited to $7.1 \mathrm{~nm}$ PV and $1.6 \mathrm{~nm}$ RMS,

Eventually, we can compare these results with the expected worst-case deformations evaluated during the design phase. Thermomechanical contribution could not be evaluated so only mechanism induced deformation are considered.

\begin{tabular}{|c|c|c|c|}
\hline & $\begin{array}{l}\text { Average measured impact } \\
\text { of mechanism on mirror } \\
\text { RWE }\end{array}$ & $\begin{array}{l}\text { Average measured impact } \\
\text { of mechanism on mirror } \\
\text { SFE }\end{array}$ & $\begin{array}{l}\text { Evaluated worst case for mirror SFE } \\
\text { (mechanism integration only) }\end{array}$ \\
\hline PV & 7.1 & 3.5 & 5.7 \\
\hline RMS & 1.6 & 0.8 & 3.1 \\
\hline
\end{tabular}

Table 3: comparison of mirror measurements and expected design worst cases

All measured values are within the expected range of mirror deformation, indicating that the conservative simulation approach was correct in that case.

\section{PAM30 PRODUCTION AND ASSEMBLY}

\subsection{Titanium parts - alpha case removal process qualification}

The use of titanium for some parts, justified by its interesting mechanical properties and heritage on CTEC previous mechanisms (for similar parts) remains demanding in terms of manufacturing process. The main issue is coming from the use of wire electro discharge machining (WEDM) for the "flexible" parts manufacturing, i.e. the actuator amplification shell and the guiding blade, at the centre of the mechanism. This manufacturing technique is required due to the parts geometry. 

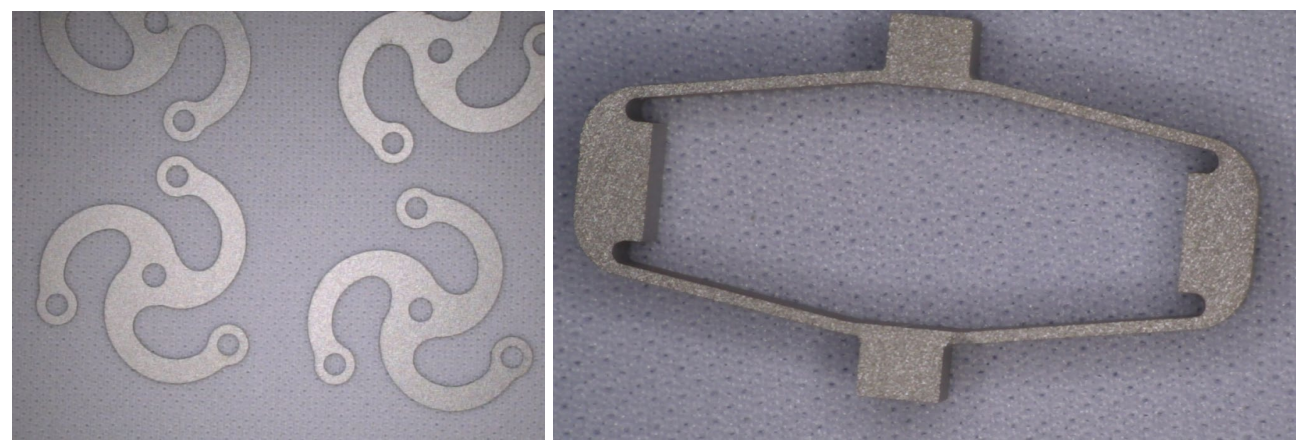

Figure 5: WEDM titanium parts, guiding blade (left) and actuator shell (right), different scale

The WEDM locally heats the material which can induce the formation of alpha-case on surface, with one of the adverse effects being a significant lifetime reduction (up to $30 \%$ according to [6]) and possibly hardly predictable failures. This alpha case, usually a few $\mu \mathrm{m}$ thick, can then be removed through proper chemical etching.

For this mechanism, the already qualified manufacturer was not compatible with the required schedule (overload), hence a backup option had to be identified and qualified. The new supplier proposed an acid etching process. The qualification process was the following:

- Machine a set of guiding blade and actuator shell, identical to flight design (supplier, process, material batch, dimensions)

- Perform an alpha case analysis before acid etching, parts in initial state, as well as an interstitial hydrogen contamination measurement.

- Pass the parts through the acid etching process, adjusted to remove the required thickness.

- Perform an analysis after the chemical etching: alpha case and interstitial hydrogen contamination.

- The process is qualified if analysis show no trace of alpha case after etching and no more than 150ppm interstitial hydrogen contamination.

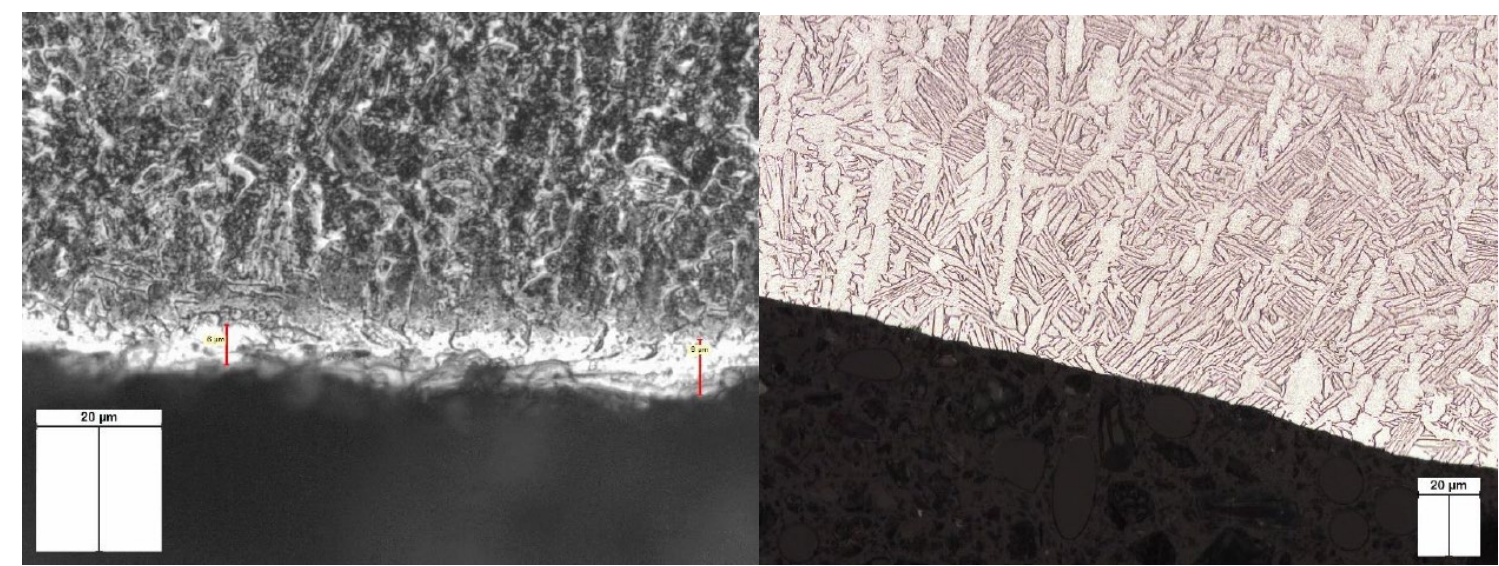

Figure 6: Alpha case analysis, before (left) and after the acid etching (right)-Optical microscope (x1000) ${ }^{\circ}$

No alpha case was found on that actuator shells, on the guiding blade however the average alpha case thickness was $8 \mu \mathrm{m}$ (6 to $11 \mu \mathrm{m}$ ). It was decided that a $15 \mu \mathrm{m}$ thickness removal through acid etching would be enough to remove the alpha case.

The final control after the acid etching indicates that the process efficiently removed the alpha case (see Figure 5), no trace was left on the part. The hydrogen contamination, that can be induced during the acid etching (penetration of hydrogen inside the material compound and local embrittlement), remained within the boundaries (86ppm for guiding blade and 26ppm for actuator shells). The process is then qualified and was used successfully to treat flight parts. 


\subsection{Piezoelectric stacks - Lot Acceptance Tests (LAT)}

As one of the critical items in the mechanism (brittle, sensitive part), the piezo stacks are dealt with a special care. One single piezo batch is procured for the project, with important quantity margins (at least $\mathrm{x} 2$ ). A LAT is then performed on 4 piezo stacks taken from this batch, prepared (SG gluing, cabling) exactly as flight piezos.

This LAT includes a thermal cycling and a lifetime tests representative to the final environment (this includes mounting them in their actuator shell) are performed. Regular basic electrical verifications (capacitance, insulation), stroke measurements are performed before and after the tests to detect any deviation. A final destructive physical analysis (DPA) is performed to inspect inner features of the piezo stacks: mainly to detect potential voids in the ceramic and electrode delamination. Piezo stacks are cut in 2 and sections are inspected.

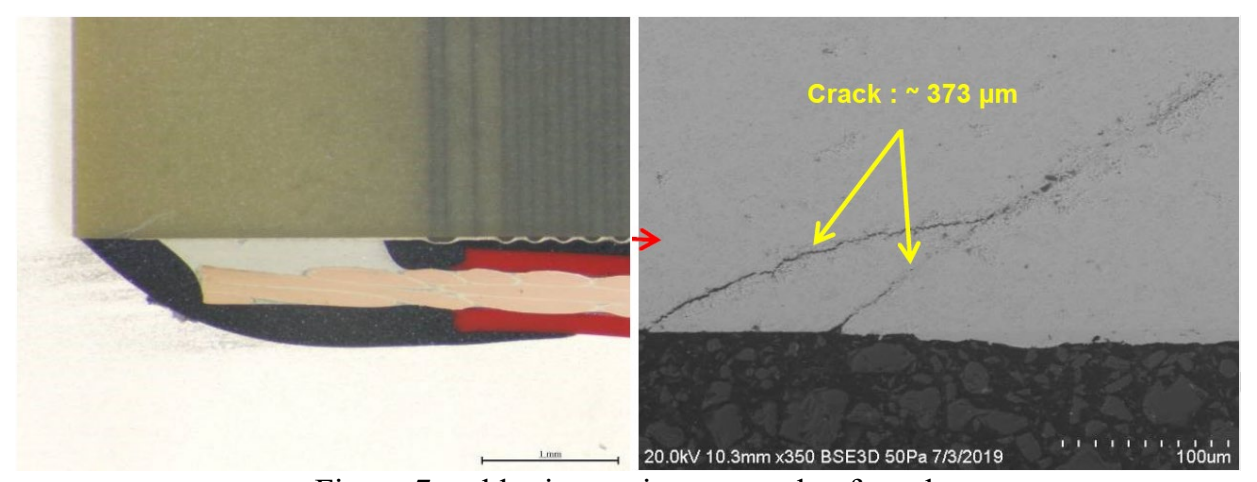

Figure 7: solder inspection, example of crack

The DPA reveals that the material is dense enough, no voids are detected. Electrodes are perfectly in place, no trace of delamination. In compliance with CTEC previous experiences, small $45^{\circ}$ cracks are detected in the vicinity of the electrodes. Those cracks are believed to be caused by the thermal expansion and shrink of the electrode during soldering. Since they are covered in epoxy securing, the cracks progression is contained.

How-ever one crack, unusually placed and lengthy, was detected. Unusual by its location, starting from piezo edge instead of from electrode, and by its length: $2.2 \mathrm{~mm}$ compared to usual few hundreds of $\mu \mathrm{m}$.

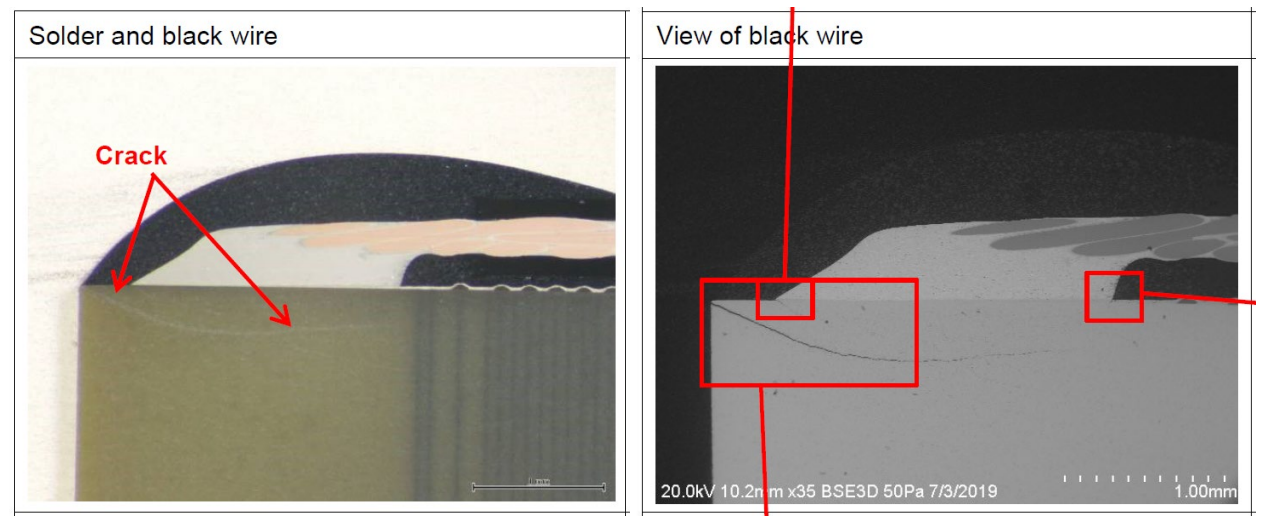

Figure 8: Piezo n³, black wire side unusual crack (length $2.2 \mathrm{~mm}$ )

Raising many questions, investigations were performed but no clear specific root cause was identified. It was found that this crack was slightly visible from the exterior and most importantly not going through the entire stack (staying near electrode, under glue). 
One possible root cause for this crack is stress concentration due to a slight mispositioning inside the actuator shell and to the selected prestress level. Simulations representing this mispositioning allowed to map the induced stress concentration and the result is visually like the observed crack pattern.
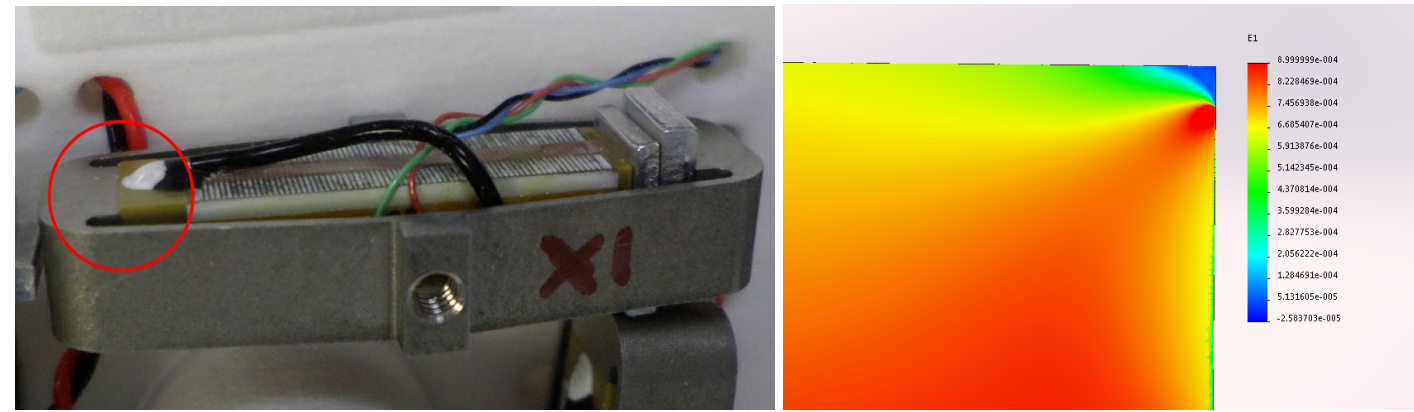

Figure 4: picture of piezo mispositioning and simulation of corresponding principal deformation map (cut view) matching crack picture

To avoid this problem, final flight actuator shell will be larger $(9 \mathrm{~mm}$ instead of $5 \mathrm{~mm})$ than the one used for the LAT, making it impossible for the piezo face to be in contact with an edge like this. Also, for precautions, all piezo stacks were inspected before assembly in flight hardware and no exterior sign of similar crack was found.

No other unusual aspects were detected, and the piezo batch was accepted for integration.

\subsection{Mechanism integration}

The main constraint of this mechanism integration is that the support bracket (black anodized aluminum part) has its interface surface (fixed to the L3 optical array) perpendicular to the mechanism base plane. This geometry is severely limiting the access options to the mechanism: for torque wrench, operator hands, pliers etc.... Hence the assembly process had to integrate this specific constraint with the unusual option to pre-assemble the actuation mechanism outside its final base plate, then transferring it onto the final support bracket. The final mechanism once assembled is presented in Figure 1.

The most sensitive part of the mechanism is its coated $\mathrm{SiC}$ mirror and many precautions were taken to protect it during the assembly. A specific cover (POM-C) was designed and was used for most assembly phases, especially to protect the mirror surface from wrench or pliers when needed. To ensure additional protection during transportation phases (models are transported several times during assembly and test phases), a transparent Plexiglas box has been designed.
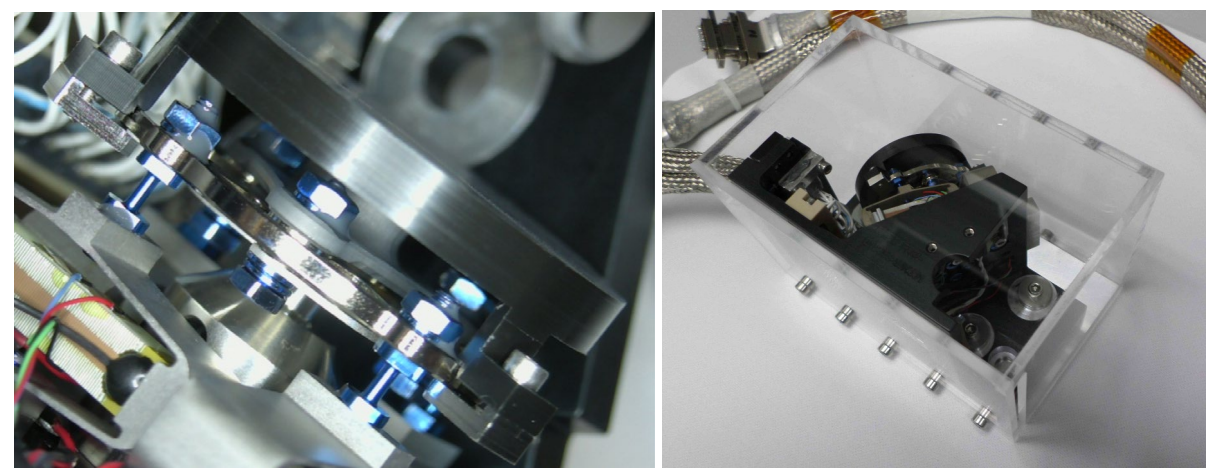

Figure 5: Mirror protection cover once installed (black part), PAM30 transportation box 


\subsection{Cables and PCB integration}

A total of 16 SG sensor wires and 8 piezo power wires had to be routed from the mechanism to the interface cables. The use of a connector on PAM30 side was not possible due to size constraints. The selected design option is an interconnection PCB, similar to what was used in CTEC previous space mechanism like the ATLID BSM ${ }^{(1)}$. The multilayer PCB provides integration process with many advantages:

- Allows pre routing of all SG bridges easily,

- Easier wire handling and more reliable interconnection (from AWG36 SG wires to AWG26 pigtail cable wire, important number of wires),

- More control of SG traces length (and impedance) to ensure a reduction of SG offset thermal drift,

- SG and piezo signals are routed through different layers, insulated and shielded from each other (ground planes in the middle of the PCB),

- Interconnection PCB was used in previous similar space projects with positive experience feedback,

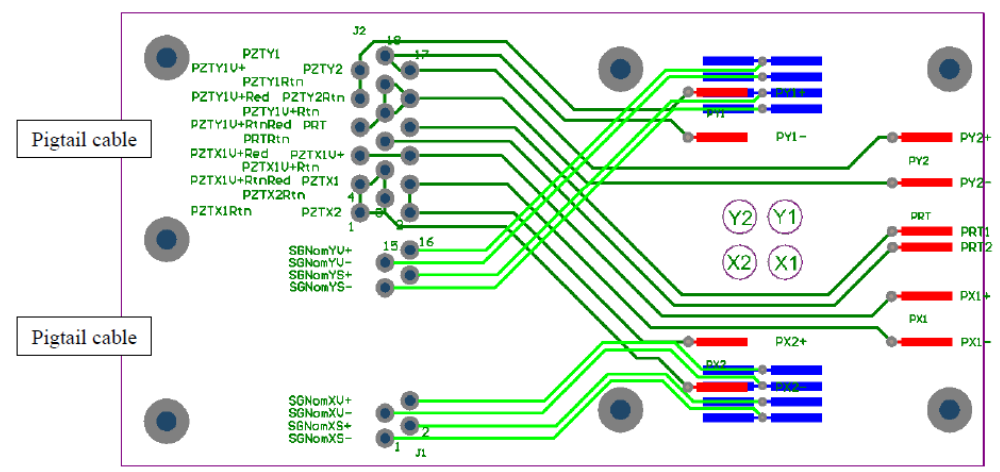

Figure 6: PAM PCB routing overview (merged layers for easier visualization)

Wires are routed from their starting point on the piezo actuators (SG and power), go through the support bracket via holes up to their dedicated PCB pad. Wire paths are regularly secured with epoxy dots. The major constraint on SG wires is to have the same length for all within each Wheatstone bridge (one for each axis) to ensure they keep the same resistance within the target range.
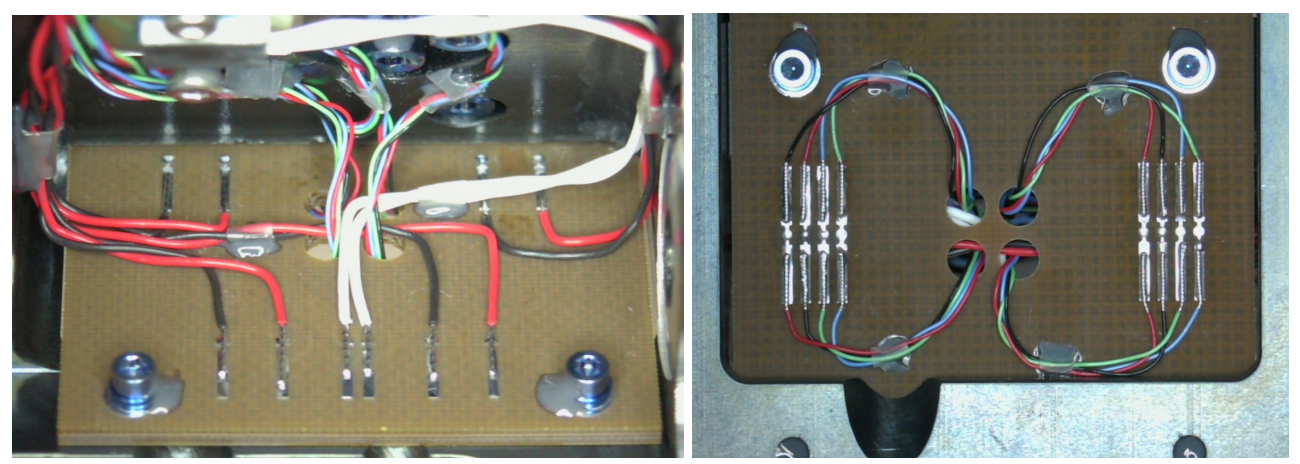

Figure 7: PAM30 cabling to interconnection PCB 


\section{QUALIFICATION AND TESTS CAMPAIGN}

\subsection{Static performances}

The 4 mechanism performances are controlled at different steps of their acceptance tests. An initial good health verification is performed to ensure that the piezo and SG parts are correctly cabled.
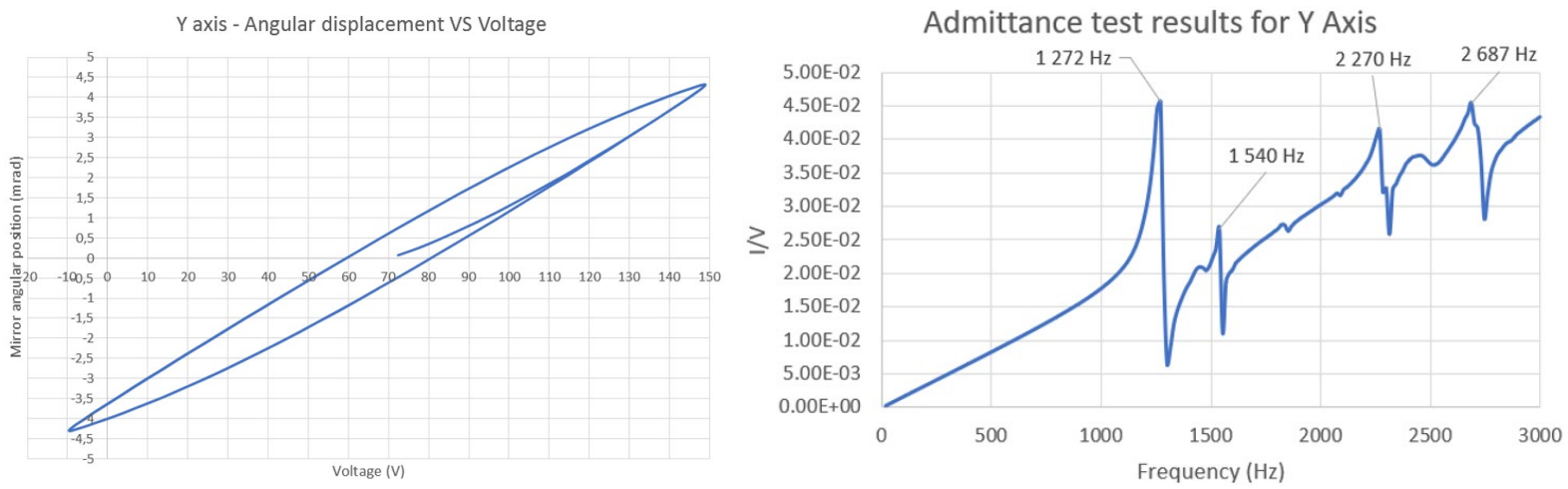

Figure 8: EQM Y axis full stroke measurement (mirror angle vs push-pull voltage) and admittance sweep for EQM Y axis - coupled resonance frequency identification.

The 4 models of the PAM30 have been tested and the results are presented in the following table.

\begin{tabular}{|c|c|c|c|c|c|}
\hline Parameter & Required value & EM & EQM & FM2 & FM3 \\
\hline \multicolumn{6}{|c|}{ PAM total stroke at ambient $(-10 /+150 \mathrm{~V})$} \\
\hline Xaxis & $>6 \mathrm{mrad}$ & $8,0 \mathrm{mrad}$ & $8,7 \mathrm{mrad}$ & $8,5 \mathrm{mrad}$ & $8,2 \mathrm{mrad}$ \\
\hline $\mathrm{Y}$ axis & $>6 \mathrm{mrad}$ & $8,6 \mathrm{mrad}$ & $8,6 \mathrm{mrad}$ & $8,5 \mathrm{mrad}$ & $8,4 \mathrm{mrad}$ \\
\hline \multicolumn{6}{|c|}{ SG response at ambient- Functional tests FTM-01-B (cf, III,8) } \\
\hline \multicolumn{6}{|l|}{ Offset } \\
\hline Xaxis & l & $-0,71 \mathrm{mrad}$ & $-0,27 \mathrm{mrad}$ & $-0,46 \mathrm{mrad}$ & $-0.097 \mathrm{mrad}$ \\
\hline Y axis & 1 & $-0,76 \mathrm{mrad}$ & $-0,35 \mathrm{mrad}$ & $0,11 \mathrm{mrad}$ & $-0.004 \mathrm{mrad}$ \\
\hline \multicolumn{6}{|l|}{ Gain } \\
\hline Xaxis & 1 & $1,57 \mathrm{mrad} / \mathrm{V}$ & $1,49 \mathrm{mrad} / \mathrm{V}$ & $1,76 \mathrm{mrad} / \mathrm{V}$ & $1.73 \mathrm{mrad} / \mathrm{V}$ \\
\hline $\mathrm{Y}$ axis & 1 & $1,38 \mathrm{mrad} / \mathrm{V}$ & $1,54 \mathrm{mrad} / \mathrm{V}$ & $1,67 \mathrm{mrad} / \mathrm{V}$ & $1.65 \mathrm{mrad} / \mathrm{V}$ \\
\hline \multicolumn{6}{|c|}{ 1st coupled resonance frequency } \\
\hline Xaxis & $>800 \mathrm{~Hz}$ & $1495 \mathrm{~Hz}$ & $1301 \mathrm{~Hz}$ & $1257 \mathrm{~Hz}$ & $1264 \mathrm{~Hz}$ \\
\hline $\mathrm{Y}$ axis & $>800 \mathrm{~Hz}$ & $1242 \mathrm{~Hz}$ & $1272 \mathrm{~Hz}$ & $1272 \mathrm{~Hz}$ & $1257 \mathrm{~Hz}$ \\
\hline \multicolumn{6}{|c|}{ PAM mass } \\
\hline Total mass & 1 & $497,2 \mathrm{~g}$ & $497,9 \mathrm{~g}$ & $495,1 \mathrm{~g}$ & $496,3 \mathrm{~g}$ \\
\hline
\end{tabular}

Table 4: static measurement results for PAM30 EM/EQM and 2 FMs

The mechanism total stroke is compliant with the specification with important margins since design anticipated the stroke loss in cold temperature. The total stroke is similar for all the models' axis, the slight variation we can observe is expected and due to piezo actuators' stroke \& stiffness variation.

SG parameters are noted for indication, only the variation of these parameters (through temperature) will matter. Note that $\mathrm{SG}$ measurements are taken after bridge output conditioning (5V excitation, 337 and 352V/V gain for $\mathrm{X}$ and $\mathrm{Y}$ ).

\subsection{Thermal vacuum testing}

The qualification campaign for the EQM included both a non-operational (NOP) and an operational (OP) thermal vacuum cycling. Target vacuum is $<10-5$ mbar. Tests A, B, C are functional tests (stroke, SG and admittance sweep). 

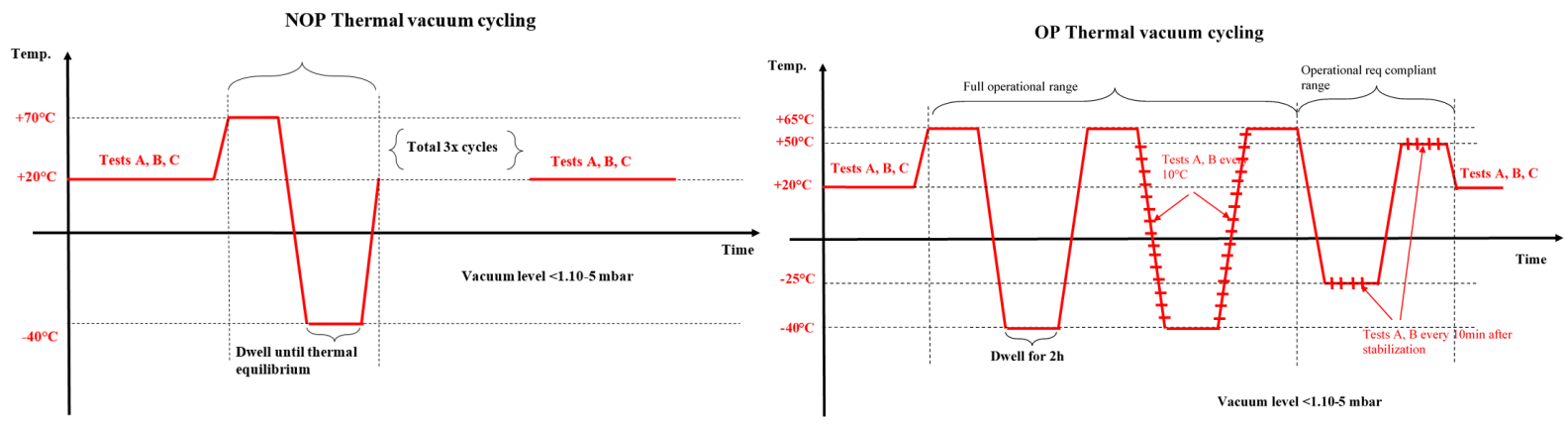

Figure 9: NOP and OP thermal vacuum cycling test schematic

The main target of the OP TVC is to characterize the evolution of PAM30 stroke (expected loss in cold) and SG performances throughout the OP temperature range. Only the results of this test will be detailed in this paper. The OP TVC consist in testing the mechanism (full stroke, SG parameters) at different temperature steps (every $10^{\circ} \mathrm{C}$ in the end to reduce total test duration).

Given that the PAM30 applied voltage was not constant during the full test (4 days with electronic and room temperature variations), the stroke result is normalized with respect to the voltage range applied, called stroke/voltage gain. The stroke results of all tested models are indicated in the following Figure 15.

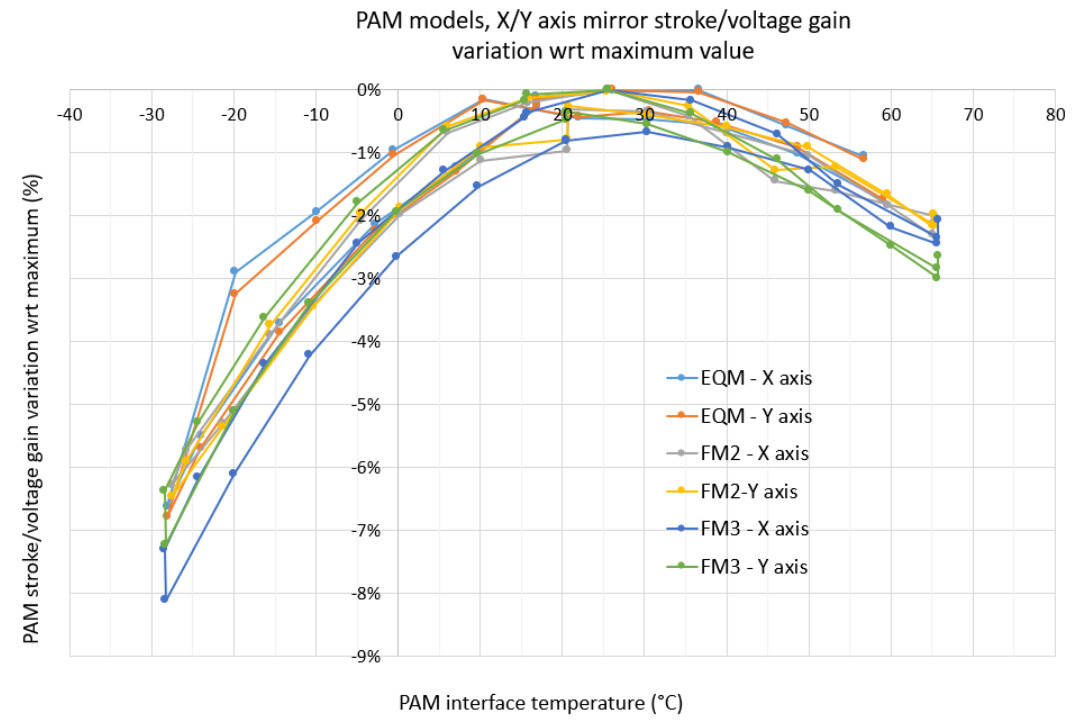

Figure 10: $\mathrm{X}$ and $\mathrm{Y}$ stroke/voltage gain through OP temperature range - EQM results

The stroke/voltage gain plots for both axes show the expected bell shape, with a maximum at ambient temperature and loss in cold and hot temperature. In the worst case, a $8 \%$ stroke loss can be noticed at $-30^{\circ} \mathrm{C}$ and around $3 \%$ loss at $+65^{\circ} \mathrm{C}$. A conservative $20 \%$ stroke loss had been expected in the design phase (adding worst cases for piezo gain, temperature loss and parts/assembly tolerances), explaining the important stroke margins we have in the end.

The other noticeable feature is the thermal hysteresis, that should not be there: it is expected for the mechanism to have the same stroke for same temperature (the stroke vs voltage hysteresis noticed is however nominal and expected). The reason for this is a probable consequence of the fact PAM30 temperature was not stabilized enough, tests were performed slightly too quickly and resulted in this thermal hysteresis. The actual temperature data is measured on the PAM30 through a thermo couple integrated on its support bracket (interface). It was not possible to bond the temperature 
sensor on the piezo ceramic to avoid any potential damage/contamination. Hence it is not possible to directly monitor the piezo temperature.

To confirm this thermal delay hypothesis, a simplified thermal-equivalent first order model of the PAM30 was created, using measured temperature time constants (between PAM30 interface and the piezo ceramics) and a bell-shaped stroke vs temperature look-up table for the piezo ceramics.

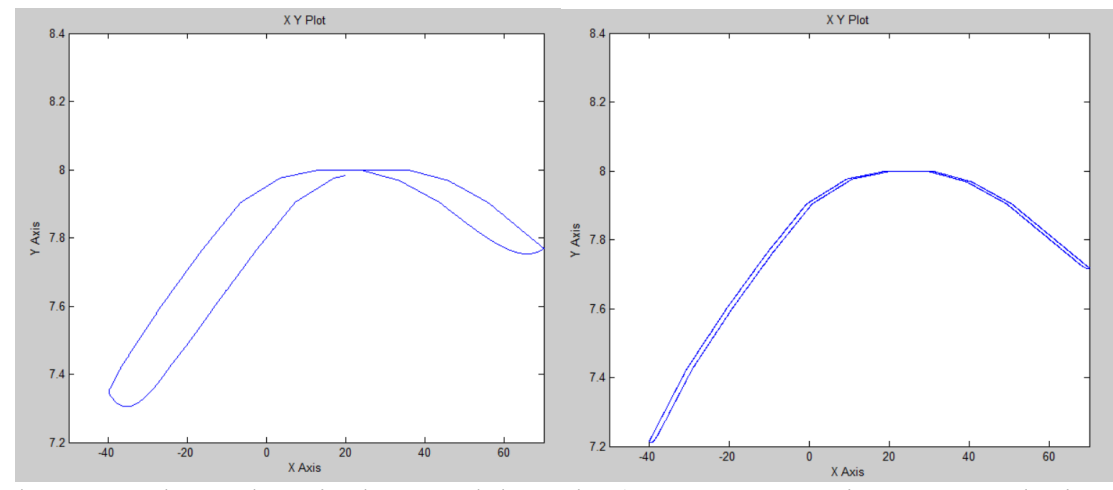

Figure 11: simplified PAM30 thermal equivalent model results (X: temperature in ${ }^{\circ} \mathrm{C}, \mathrm{Y}$ : stroke in $\left.\mathrm{mrad}\right)$, left: $10 \mathrm{~h}$ cycle duration (close to what was tested), right: 100h cycle duration where hysteresis is greatly reduced.

The model can recreate this hysteretic behavior, which appear to be greatly reduced after greatly increasing test duration (x10). However, given that the measurements cannot be automated, an operator has to be there at each step (hence excluding nights and weekends). The EQM test already lasted 3 days and a 30 day tests is not possible. Options need to be found to allow the OP TVC test automation $\&$ data acquisition.

SG parameters (offset and gain) were also measured for each temperature steps, the results for EQM X-axis are the following:

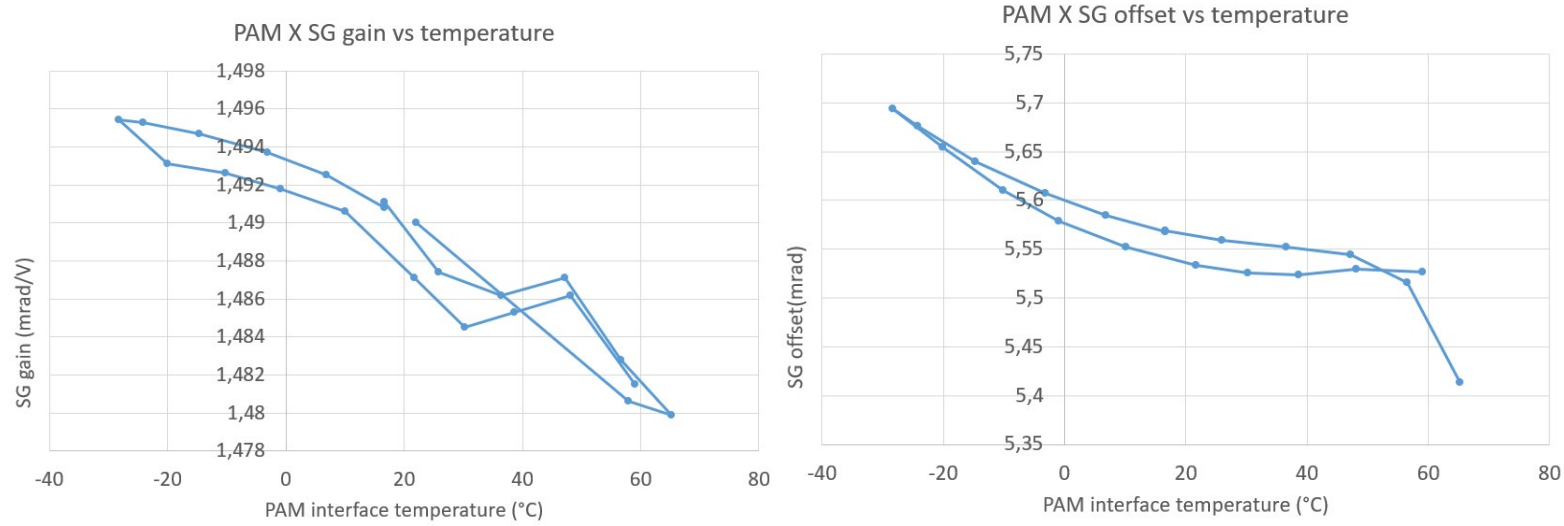

Figure 12: EQM X axis SG gain vs temperature (left), EQM X axis SG offset vs temperature (right)

The results are also affected by the piezo temperature delay. As anticipated, the SG gain is quite stable with less than 1\% variation through the temperature range. The offset is directly impacted by the mechanism thermomechanical excursion, which was measured at $0.4 \mathrm{mrad}$ total ( 0.2 to $0.4 \mathrm{mrad}$ on X and Y SG offset).

\subsection{Vibrations verification}

All models except EM are planned to go through vibration tests. The tests consist in a random vibration verification as well as a shock test, for each axis. A low-level frequency sweep is performed before and after each test (to assess potential modal landscape modification). 
Regarding the random vibration test, the initial preliminary levels for which the mechanism was justified were of $10 \mathrm{grms}$ $\left(20-2000 \mathrm{~Hz}\right.$ span with a 50 to $800 \mathrm{~Hz} 0.08 \mathrm{~g}^{2} / \mathrm{Hz}$ plateau). Later in the project, when the updated and refined DSOC full system vibration simulations were performed, the specified levels at PAM30 interface location had to be increased. The final random vibration levels are much higher and follow the hereunder power spectral density (PSD):

\begin{tabular}{|c|c|c|c|c|c|}
\hline \multicolumn{2}{|c|}{ X -Axis Test Spec } & \multicolumn{2}{c|}{ Y-Axis Test Spec } & \multicolumn{2}{c|}{ Z-Axis Test Spec } \\
\hline FREQ(Hz) & $\operatorname{ASD}\left(\mathbf{g}^{\mathbf{2}} / \mathbf{H z}\right)$ & FREQ(Hz) & $\operatorname{ASD}\left(\mathbf{g}^{\mathbf{2}} / \mathbf{H z}\right)$ & FREQ(Hz) & $\mathbf{A S D}\left(\mathbf{g}^{\mathbf{2}} \mathbf{H z}\right)$ \\
\hline 20 & 0.100 & 20 & 0.1 & 20 & 0.1 \\
\hline 50 & 3.500 & 50 & 3.5 & 50 & 3.5 \\
\hline 150 & 3.500 & 395 & 3.5 & 340 & 3.5 \\
\hline 2000 & 0.010 & 2000 & 0.01 & 2000 & 0.01 \\
\hline \multicolumn{2}{r|}{$\mathbf{G r m s}=\mathbf{2 8 . 0 3}$} & \multicolumn{2}{c|}{$\mathbf{G r m s}=\mathbf{4 2 . 0}$} & \multicolumn{2}{c|}{$\mathbf{G r m s}=\mathbf{3 9 . 5}$} \\
\hline
\end{tabular}

Table 5: final PSD for PAM random vibration test

Updated mechanism simulation showed that those new levels remain acceptable, regarding mechanical stress in the parts. The cables and wires, however, are more affected and are raised interrogations regarding the updated vibration levels. Wire cabling and secured routing (with glue) upgrades were tested to make sure there is no risk with the wires (mainly by reducing wires free length) but were not implemented in the final models.
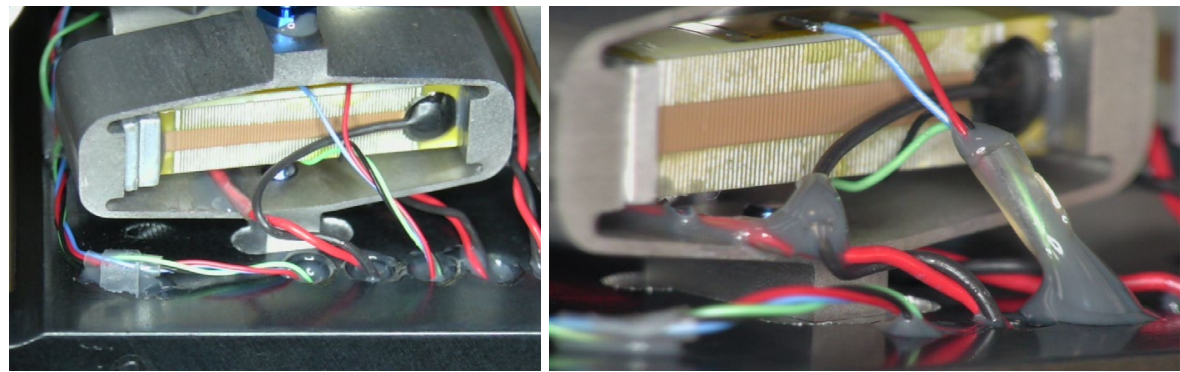

Figure 13: overview of SG wires, initial configuration (left) and reinforced cabling option (right)

The full level vibration tests have been passed with success on the EQM, FM2 and FM3.

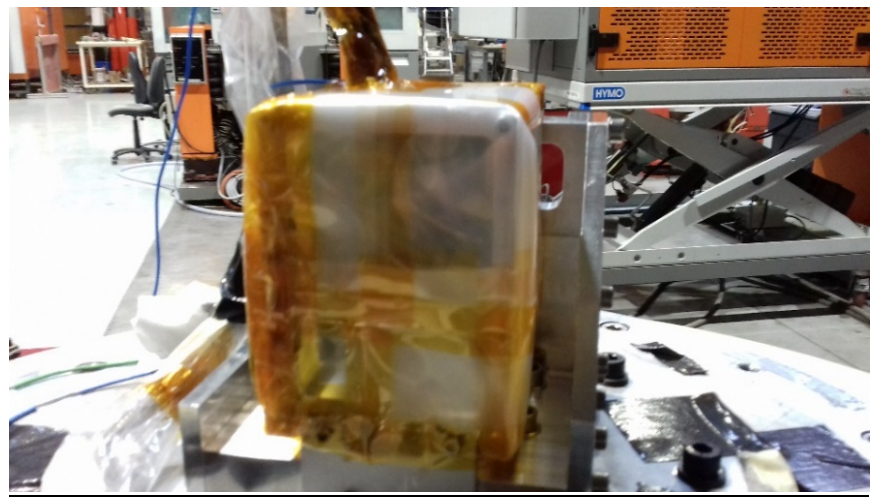

Figure 19: Clean packaged PAM30 on the shaker for vibration test

\subsection{Mechanism delivery status}

All the models have been delivered to L3-Harris in July 2020. Nevertheless, FM1 has been damaged during the subcontracted bake-out process. The failure in that bake out process was to use a 200 bars N2 tank to return to atmospheric pressure inside the vacuum chamber. A default in the N2 Tank outlet regulator generated an over pressure 
inside the chamber and the explosion of its door leading to the projection of the PAM30 on the ground and the breaking of its $\mathrm{SiC}$ mirror. Despite the breaking of its mirror, the PAM30 was still able to operate at low voltage. A new model FM1, called FM3, had to be fully assembled and tested using spare and new parts in short term.

\section{CONCLUSION}

In this paper, the development, the procurement, the assembly and the tests of a novel dual axis tilt PAM30 mechanism have been detailed. The mirror integration verification method has been explained and the comparison with actual measurements indicates that the results were quite reliable for this case.

The test results have been presented and indicates that the PAM30 mechanism have been working as intended. Some measurement artifacts on the OP TVC (thermal hysteresis) have been investigated and some improvements have been implemented for FM tests.

The cable wiring has been reinforced to ensure the mechanism survival to the random vibration and shocks with increased levels.

\section{ACKNOWLEDGMENT}

The authors want to thank all partners involved, among others: Marshall Bernklow and Richard Aigbekean from L3Harris-SSG, as well as Daniel McDonald and Joseph Kovalik from JPL-Optical-Communication-Laboratory for their support during the project.

\section{REFERENCES}

1. R. Le Letty, F. Barillot, H. Fabbro, F. Claeyssen, Ph. Guay, L. Cadiergues, Miniature Piezo Mechanisms for Optical and Space applications Proc ACTUATOR Conf, Pub. Messe Bremen (G), June 2004, pp 177-180

2. E. Prevost, A. Weickman, S. Belmana, F. Bourgain, O. Sosnicki, F. Claeyssen, Beam Steering Mechanism For Earthcare Atmospheric Lidar Instrument Atlid - An Ultra-Stable Piezoelectric Tip Tilt Mechanism, Proc. ICSO, Biarritz, Oct. 2016

3. F. Claeyssen, T. Maillard, O. Sosnicki, F. Barillot, A.Pages, C.Belly, A.Bataille, M.Logeais, G.Aigouy, T.Porchez, F. Bourgain, Beam Steering Mirrors from space applications to optronic solutions, Proc. OPTRO Conf, Paris, Feb. 2018

4. F. Claeyssen, K. Benoit, G. Aigouy, T.Maillard, M. Fournier, Olivier Sosnicki, Large-Stroke Fast Steering Mirror For Space Free-Space Optical Communication, Proc. OPTRO Conf, Paris, Feb. 2020

5. F. Bourgain, O. Sosnicki, C. Belly, F. Barillot, F. Claeyssen, An Improved Accurate Beam Steering Piezoelectric Mechanism for ATLID Instrument, Proc. Actuator 2014 Vol. pp. 293-296

6. T. Mower., Degradation of titanium $6 \mathrm{Al}-4 \mathrm{~V}$ fatigue strength due to electrical discharge machining, International Journal of Fatigue, vol.64, pp 84-96 